\title{
Epiretinal membrane surgery for combined hamartoma of the retina and retinal pigment epithelium: role of multimodal analysis
}

This article was published in the following Dove Press journal:

Clinical Ophthalmology

18 January 2013

Number of times this article has been viewed

\author{
Claudia Bruè \\ Andrea Saitta \\ Michele Nicolai \\ Cesare Mariotti \\ Alfonso Giovannini \\ Ophthalmology, Department \\ of Neuroscience, Marche Polytechnic \\ University, Ancona, Italy
}

Background: The purpose of this study was to evaluate the role of spectral domain optical coherence tomography (SD-OCT), MP-1 microperimetry, and fundus autofluorescence imaging for planning surgical procedures in combined hamartomas of the retina and retinal pigment epithelium (CHR-RPE) and following epiretinal membrane removal.

Methods: In an interventional retrospective case series, six consecutive subjects with CHRRPE underwent vitrectomy and epiretinal membrane peeling, with 4 years of follow-up. Each underwent complete ophthalmic examination, including best corrected visual acuity, fundus examination, fundus fluorescein angiography, SD-OCT, MP-1, and fundus autofluorescence at one, 6,12 , and 48 months.

Results: Six eyes from six subjects with CHR-RPE were studied (mean age $31 \pm 14$ years). All patients were phakic and five were male (83.3\%). Lesions were unilateral, ie, three macular, two juxtapapillary and macular, and one pericentral. Preoperative best corrected visual acuity was $0.3 \pm 0.08$ Snellen, with significant improvement to $0.9 \pm 0.17$ Snellen $(P=0.001)$ at 4 years of follow-up. Mean retinal sensitivity within the central $20^{\circ}$ field improved from $16.6 \pm 1.84 \mathrm{~dB}$ to $18.8 \pm 0.96 \mathrm{~dB}(P=0.07)$. There was also a statistically significant reduction in the visual defect $(P=0.04)$. SD-OCT demonstrated that the epiretinal membranes were completely removed in all but one patient, with significantly decreased macular edema on follow-up at one, 6,12 , and 48 months $(P=0.001)$. A positive correlation was shown between preoperative macular sensitivity and postoperative best corrected visual acuity. Fundus autofluorescence demonstrated a block in background autofluorescence at the site of the lesion, and hyperautofluorescence at the edematous retina overlain by the epiretinal membrane.

Conclusion: Surgery is an effective treatment for CHR-RPE. SD-OCT, fundus autofluorescence, and MP-1 are valuable and noninvasive tools to guide surgical procedures for CHR-RPE. To the best of our knowledge, this study represents the first use of MP-1 in CHR-RPE in conjunction with SD-OCT and fundus autofluorescence imaging for better guided surgery as well as anatomical and functional prognosis.

Keywords: vitrectomy, epiretinal membrane, combined hamartoma of the retina and retinal pigment epithelium

\section{Introduction}

Combined hamartoma of the retina and retinal pigment epithelium (CHR-RPE) are relatively uncommon benign tumors. They comprise a pigmented elevated mass that is commonly connected to the epiretinal membrane, which causes retinal distortion and significant consequent loss of vision. ${ }^{1}$ The use of surgery in the management of CHR-RPE remains controversial. Only a few reports have described the outcomes of vitrectomy and epiretinal membrane peeling. ${ }^{2-4}$ Here, to the best of our knowledge, 
we describe for the first time the use of MP-1 microperimetry, spectral domain optical coherence tomography (SD-OCT), and fundus autofluorescence imaging for guiding the surgical procedure and as a prognostic tool.

\section{Materials and methods}

The study design complied with the Health Insurance Portability and Accountability Act of 1996, and followed the tenets of the Declaration of Helsinki. Informed consent was obtained from the subjects for evaluation of their images and review of their charts. Six consecutive subjects with eyes exhibiting CHR-RPE were included following their examination in the Department of Ophthalmology at the Polytechnic University of Ancona, Italy, from May 2008 to September 2012. Subjects with concurrent ocular pathology, such as isolated retinal hamartomas, astrocytic hamartoma, tuberous sclerosis, congenital hyperplasia of the retinal pigment epithelium, and choroidal nevus, were excluded from the study. Patients showing pseudophakic bullous keratopathy were also excluded.

Each subject underwent a complete ophthalmic examination, which included measurement of their best corrected visual acuity and intraocular pressure, biomicroscopic examination, and fundus evaluation. They also underwent fluorescein angiography, SD-OCT using a Spectralis confocal scanning laser ophthalmoscope (Heidelberg Engineering Inc, Dossenheim, Germany), MP-1 examination (Nidek, Vigonza, Italy), and fundus autofluorescence.
These examinations were carried out at the preoperative baseline, and then at one month, 6 months, one year, and 4 years postoperatively.

Diagnosis of CHR-RPE followed a comprehensive ophthalmic examination, fundus photography, and SD-OCT for consideration of the surgical strategy. Criteria for surgery included progressive loss of vision, presence of an epiretinal membrane, and macular distortion. Standard three-port, 25-gauge, pars plana core vitrectomy (Constellation Vision System, Alcon Laboratories Inc, Fort Worth, TX), removal of the posterior hyaloids, and peeling of the glial component of the CHR-RPE (internal limiting membrane forceps Grieshaber Revolution ${ }^{\circledR}$ DSP 25-gauge) were achieved following the preoperative OCTguided surgical plane. No tamponade was used.

Surgical success was determined as stabilization or improvement of visual acuity, retinal sensitivity at MP-1, and re-establishment of macular architecture according to fundus photography, SD-OCT, and fundus autofluorescence. Statistical analysis was performed using the Statistical Package for the Social Sciences version 16.0 (SPSS Inc, Chicago, IL).

\section{Results}

Six consecutive patients (for six eyes) presented with CHR-RPE (Table 1). The mean age of the patients was $31 \pm 14$ years and five were male $(83.3 \%)$. The patients all had a negative medical history, with no systemic manifestations or pathology associated with CHR-RPE or

Table I Optical coherence tomography and preoperative and postoperative clinical findings in the six patients affected by combined hamartoma of the retina and retinal pigment epithelium

\begin{tabular}{|c|c|c|c|c|c|c|c|c|}
\hline Patient & $\begin{array}{l}\text { Vitreoretinal } \\
\text { traction }\end{array}$ & ERM & $\begin{array}{l}\text { Retinal } \\
\text { folds }\end{array}$ & $\begin{array}{l}\text { Retinal } \\
\text { disorganization }\end{array}$ & $\begin{array}{l}\text { Photoreceptor } \\
\text { attenuation }\end{array}$ & $\begin{array}{l}\text { Retinal } \\
\text { thickness }\end{array}$ & BCVA & $\begin{array}{l}\text { Retinal } \\
\text { sensitivity }\end{array}$ \\
\hline \multicolumn{9}{|l|}{ I } \\
\hline Preoperative & Yes & Yes & Yes & Yes & Yes & $646 \mu \mathrm{m}$ & 0.3 & $14.2 \mathrm{~dB}$ \\
\hline Postoperative & No & Yes & No & No & Yes & $538 \mu \mathrm{m}$ & 0.6 & $16.9 \mathrm{~dB}$ \\
\hline \multicolumn{9}{|l|}{2} \\
\hline Preoperative & Yes & Yes & Yes & Yes & Yes & $625 \mu \mathrm{m}$ & 0.3 & $15.1 \mathrm{~dB}$ \\
\hline Postoperative & No & No & No & No & Yes & $270 \mu \mathrm{m}$ & 1.0 & $18.8 \mathrm{~dB}$ \\
\hline \multicolumn{9}{|l|}{3} \\
\hline Preoperative & Yes & Yes & Yes & Yes & No & $597 \mu \mathrm{m}$ & 0.4 & $18.1 \mathrm{~dB}$ \\
\hline Postoperative & No & No & No & No & No & $300 \mu \mathrm{m}$ & 1.0 & $19.4 \mathrm{~dB}$ \\
\hline \multicolumn{9}{|l|}{4} \\
\hline Preoperative & Yes & Yes & Yes & Yes & Yes & $625 \mu \mathrm{m}$ & 0.2 & $16.5 \mathrm{~dB}$ \\
\hline Postoperative & No & No & No & No & No & $398 \mu \mathrm{m}$ & 0.9 & $19.4 \mathrm{~dB}$ \\
\hline \multicolumn{9}{|l|}{5} \\
\hline Preoperative & Yes & Yes & No & Yes & Yes & $630 \mu \mathrm{m}$ & 0.2 & $16.7 \mathrm{~dB}$ \\
\hline Postoperative & No & No & No & No & No & $402 \mu \mathrm{m}$ & 0.9 & $19.0 \mathrm{~dB}$ \\
\hline \multicolumn{9}{|l|}{6} \\
\hline Preoperative & Yes & Yes & Yes & Yes & Yes & $733 \mu \mathrm{m}$ & 0.4 & $19.1 \mathrm{~dB}$ \\
\hline Postoperative & No & No & No & No & No & $469 \mu \mathrm{m}$ & 1.0 & $19.3 \mathrm{~dB}$ \\
\hline
\end{tabular}

Abbreviations: ERM, epiretinal membrane; BCVA, best corrected visual acuity. 
previous ocular disease. Mean follow-up was $23 \pm 16.28$ (range 12-48) months. Mean preoperative intraocular pressure was $14 \pm 2 \mathrm{mmHg}$ and biomicroscopy of the anterior segment was normal. All patients were phakic.

Ophthalmoscopic examination revealed the CHR-RPE lesions, which were gray-brown, unilateral, unifocal and formed by glial tissue. Three of the patients showed macular lesions (50\%) (Figure 1A), while two were juxtapapillary and macular $(33.3 \%$, Figure $1 \mathrm{~B})$ and one was peripheral (16.7\%, Figure 1C).

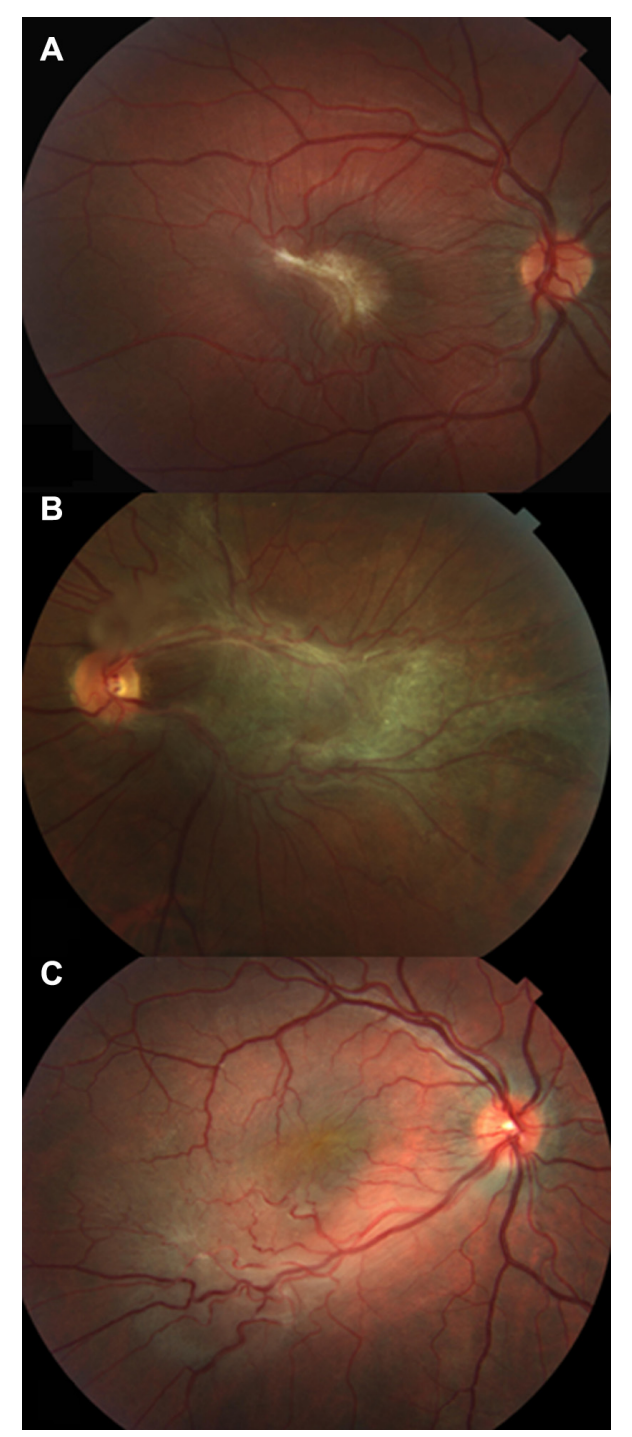

Figure I (A) Photograph of the fundus of the right eye in an 18-year-old girl who complained of metamorphopsia and reduced visual acuity, which was due to retinal folding corresponding to the epiretinal membrane underlying CHR-RPE in the macula. (B) Photograph during preoperative fundus examination of a 52-year-old man affected by CHR-RPE, showing a slight elevation on the optic disc and macula, retinal vessel tortuosity, hyperpigmentation, and extensive epiretinal membrane. (C) Photograph of preoperative peripheral CHR-RPE with epiretinal membrane in a 33-year-old man, showing retinal vessel tortuosity and visual distortion.

Abbreviation: CHR-RPE, combined hamartoma of the retina and retinal pigment epithelium.
A preretinal membrane was detected in all six patients using SD-OCT (Figure 2A and B). The preretinal membrane appeared with peak configuration due to anteroposterior vitreoretinal traction in all six patients $(100 \%)$, while retinal folds were detected in four patients $(66.7 \%)$. A thickened disorganized retina with a hyper-reflective surface, clear margins of the lesion, and deep shadowing were identified in all patients (Figure 2B) which, in conjunction with the partly pigmented lesion, led us to rule out macular pucker. The retinal mass showed loss of the clear retinal layers in all patients.

The mean foveal thickness, regardless of location of the epiretinal membrane, was $645.2 \pm 46.58 \mu \mathrm{m}$ preoperatively and $395 \pm 83.50 \mu \mathrm{m}$ postoperatively. Attenuation of the photoreceptor layer was observed in five $(83.3 \%)$ of the patients (Figure 2A and B, arrows). Macular edema was present in five patients $(83.3 \%)$, while no subretinal fluid was observed with SD-OCT. SD-OCT was used to detect the surgical plane, and to localize the point of epiretinal membrane adhesion and separation from the retina (Figure 2B, arrowheads).

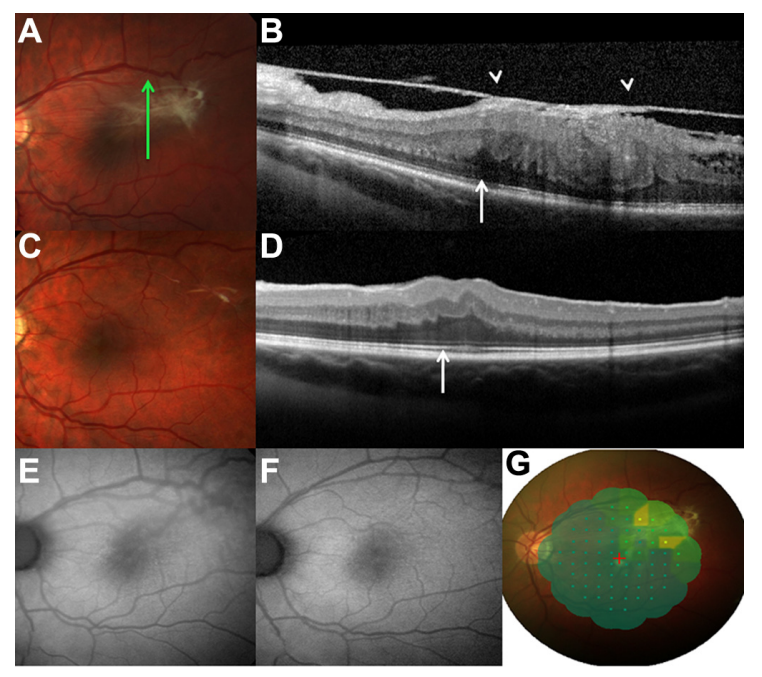

Figure 2 (A) Photograph during the preoperative fundus examination of a 26-yearold man, with slight elevation at the temporal macula, and hyperpigmentation and epiretinal membrane with retinal vessel tortuosity. (B) Preoperative spectral domain optical coherence tomography analysis showing disorganization of the normal retinal layers, loss of inner-outer segment photoreceptor junction (arrow), an elevated lesion with high reflectivity of the inner retina, hyporeflective shadowing of the tissue, and epiretinal membrane with vitreous traction (arrowheads). (C) Photograph 4 years postoperatively, where the epiretinal membrane has disappeared and the retinal vessel tortuosity has been relieved. (D) Spectral domain optical coherence tomography analysis at 4 years shows a normal appearance of the retinal layers and a clear inner-outer segment photoreceptor junction. (E) Preoperative fundus autofluorescence demonstrates a block in the background autofluorescence at the site of the lesion, and a slightly hyperautofluorescent area with the slightly indistinct appearance of the macular edema and epiretinal membrane. (F) At 4 years after surgery, fundus autofluorescence imaging detected normal autofluorescence of the macular region, with a reduction in retinal vessel tortuosity. (G) MP-I highlighting lower retinal sensitivity corresponding to the area with higher adherence of the epiretinal membrane to the combined hamartoma of the retina and retinal pigment epithelium lesion preoperatively. 
Postoperatively, the patients showed minor vascular tortuosity and total removal of the glistening epiretinal membrane in all cases except one (Figure 2C), in which the membrane was too closely adherent to the retina to be peeled without complications. Reduced dye leakage at the late phases was seen with fluorescein angiography at the last follow-up, in comparison with the preoperative baseline, for all of the eyes.

In the postoperative examination at one month, SD-OCT showed that all of the components of the epiretinal membranes had been completely removed (Figure 2D), with integrity of the junction between the inner and outer segments of the photoreceptors evident in three cases (50\%, Figure 2D). Restoration of physiological retinal morphology and a decrease in underlying retinal edema was seen in all patients, except for one at 4 years. Patients who had shown integrity of the junction between the inner and outer segments of the photoreceptors at the preoperative SD-OCT had better best corrected visual acuity and isoautofluorescence on fundus autofluorescence at the last follow-up. SD-OCT highlighted the cleavage plane of the membrane. Fundus autofluorescence demonstrated a block in the background autofluorescence at the site of the lesion and a slightly hyperautofluorescent area with a fuzzy appearance in the case of macular edema and epiretinal membrane (Figure 2E). Six months after surgery and up to the last follow-up at 48 months, fundus autofluorescence imaging revealed normal autofluorescence of the macular region, with a reduction in vessel tortuosity (Figure 2F).

Initial symptoms in all patients included blurred vision. Preoperative best corrected visual acuity was $0.3 \pm 0.08$ Snellen, and significant improvement in best corrected visual acuity was detected at 4 years follow-up $(0.9 \pm 0.17$ Snellen, $P=0.001)$. At the same time, mean retinal sensitivity within the central $20^{\circ}$ field improved from the preoperative measure of $16.6 \pm 1.84 \mathrm{~dB}$ to $18.8 \pm 0.96 \mathrm{~dB}$ at the last follow-up $(P=0.07)$. We also noted that although the patient group was small, overall it was apparent that patients with lower sensitivity preoperatively had a worse visual outcome postoperatively. At the preoperative baseline, the mean sensitivity within the area of tight adhesion of the epiretinal membrane overlying the tumor was markedly reduced (Figure 2G). Compared with before surgery, there was a statistically significant reduction in the visual defect detected at the last follow-up at 4 years $(P=0.04)$. There have been no serious adverse events recorded to date, ie, at the 4-year follow-up.

\section{Discussion}

CHR-RPE is a peculiar tumor at the optical disc and posterior pole that affects not only the retina and retinal pigment epithelium, but also has an anomalous vitreoretinal interface, with consequent retinal traction and folding. ${ }^{1}$ To date, the surgical management of CHR-RPE remains controversial in terms of recovery of visual acuity.

In 1984, the Macular Society reported on a series of 60 patients affected by CHR-RPE, of whom only three underwent epiretinal membrane peeling. ${ }^{5}$ All of these subjects obtained relief from macular distortion, although only one showed improved visual acuity postoperatively, from $20 / 200$ to $20 / 40$. The authors hypothesized that if the membranes were linked tightly to the tumor vitreous, then surgery would fail to recover the lesion. ${ }^{5}$ Indeed, peeling of a membrane intrinsic to the dysplastic retina can damage the retinal fiber layer and Muller cells. Gass ${ }^{6}$ indicated that the surface glial membrane causing the retinal distortion is often an integral part of the tumor, which can mean that it is difficult or impossible to strip the membrane and that there is little chance of recovering central vision. ${ }^{5}$ Stallman ${ }^{3}$ described a 10-year-old girl who underwent successful vitrectomy and epiretinal membrane peeling, with histopathological examination of the specimen. The glistening membrane did not show any hallmarks that characterized this membrane as having components intrinsic to the retina, and the ultrastructural composition was analogous to an idiopathic epiretinal membrane. Stallman ${ }^{3}$ speculated that the membrane is not interwoven within the dysplastic retina, and that this lesion could be a combined hamartoma of the retina, retinal pigment epithelium, and vitreous. There are no established criteria to determine how intrinsic the membrane is to the retina or to the cortical vitreous. An important role in this regard can be played by SD-OCT. In all of our cases, SD-OCT demonstrated deep shadowing with a normal adjacent retina and a hyper-reflective line overlying the lesion. This suggests that the membrane is extrinsic to the retina. SD-OCT was useful for defining the exact location of the membrane and its cleavage plane.

Considering the difficult dissection of the epiretinal membrane in younger subjects, with a consequent worse surgical outcome compared with adults, it may well be that the posterior vitreous is more tightly adherent to the basal lamina of the internal limiting membrane in young patients, than in older ones. Shields et $\mathrm{al}^{7}$ described SD-OCT findings in 11 patients who were younger than those in our present series, and concluded that the epiretinal membrane is a clear 
entity separate from the CHR-RPE. Our OCT findings are consistent with theirs. We would speculate that the pigmented component of the CHR-RPE can migrate in the vitreous cavity and induce a reaction promoting formation of the epiretinal membrane that is responsible for the tractional action and the architectural disorganization of the retina.

McDonald et $\mathrm{al}^{8}$ described two young adult patients with CHR-RPE and epiretinal membrane in whom visual acuity did not improve following surgery. They attributed this unfavorable outcome to longstanding loss of visual acuity and the presence of marked macular edema. Sappenfield and Gitter ${ }^{9}$ reported improvement in visual acuity after surgical peeling of the epiretinal membrane in a young woman. Subsequently, other studies have achieved visual benefits from vitreous surgery. More recently, Shields et al ${ }^{10}$ reported a large series of 79 eyes affected by CHR-RPE. Four of these underwent pars plana vitrectomy and epiretinal membrane peeling, including one with vitreous hemorrhage. In three of these eyes, the dysplastic disorder was macular and had a tractional component. Although the preoperative visual acuity was not available in one of these cases, it improved in one patient and stabilized in another. Xiao et $\mathrm{al}^{2}$ described five patients with CHR-RPE who underwent vitrectomy and epiretinal membrane peeling. Visual acuity improved in three of the patients and remained unchanged in two. The lack of improvement in visual acuity after surgery in one of these last two cases was due to a residual macular membrane that was too tightly adherent to be removed completely. The second of these last two cases was a young boy whose vision did not improve although the membrane was completely removed, probably because of lack of treatment for amblyopia.

The visual acuity in our series of patients improved significantly in each case, which is probably because it was possible to remove the epiretinal membranes successfully in all cases except one. In this exception, the remaining component was extramacular and consequently did not affect the functional outcome. At the same time, the SD-OCT lesions were more recent than 6 months, and restoration of the inner-outer segment junctions in most cases provided for better visual outcomes.

Fundus autofluorescence imaging in CHR-RPE has been described only once, and here we have contributed additional information on its use in this setting. The fundus autofluorescence in our series detected a fuzzy hyperautofluorescent area in the case of macular edema. Because the CHR-RPE contains melanin, a hypoautofluorescent area that corresponds to this heavily pigmented lesion has a rationale. This pigment in the outer segments of the photoreceptors can result in a fuzzy hyperautofluorescent area overlying the lesion in the case of the epiretinal membrane, macular edema, and attenuation of the photoreceptor area. This raises the possibility of a lack of pigment to absorb the excitation light of the fundus autofluorescence imaging, which might lead to regional hyperautofluorescence. It has also been hypothesized ${ }^{11}$ that increased fundus autofluorescence might arise from extracellular fluid that can include autofluorescent proteins, such as the retinoids. The epiretinal membrane might displace this macular pigment in the retina, with consequent hyperautofluorescence.

Use of MP-1 in CHR-RPE for our series allowed precise localization of the retinal area with low sensitivity, which corresponds to tighter epiretinal membrane adherence. In addition, MP-1 demonstrated that patients with lower sensitivity preoperatively tend to have a worse visual outcome postoperatively. Thus, surgery for epiretinal membrane in CHR-RPE should be considered before severe worsening of retinal sensitivity because the preoperative severe low retinal sensitivity may not be completely resolved.

In conclusion, SD-OCT, fundus autofluorescence imaging, and MP-1 are useful and noninvasive tools that can provide important information regarding the vitreoretinal interface of CHR-RPE, and can define the surgical plane and provide a visual prognosis.

\section{Disclosure}

The authors report no conflicts of interest in this work.

\section{References}

1. Elliot D, Schachat AP. Combined hamartoma of the retina and retinal pigment epithelium. In: Ryan SR, editor. Retina. St Louis, MO: Mosby Ed; 2001.

2. Xiao Z, Fangtian D, Rongping D, Weihong Y. Surgical management of epiretinal membrane in combined hamartomas of the retina and retinal pigment epithelium. Retina. 2010;30(2):305-309.

3. Stallman JB. Visual improvement after pars plana vitrectomy and membrane peeling for vitreoretinal traction associated with combined hamartomas of the retina and retinal pigment epithelium. Retina. 2002;22(1):101-104.

4. Cohn AD, Quiram PA, Drenser KA, Trese MT, Capone A. Surgical outcomes of epiretinal membranes associated with combined hamartoma of the retina and retinal pigment epithelium. Retina. 2009;29(6):825-830.

5. Schachat AP, Shields JA, Fine SL, et al. Combined hamartoma of the retina and retinal pigment epithelium. Ophthalmology. 1984;91(12):1609-1615.

6. Gass JDM. An unusual harmatoma of the pigment epithelium and retina simulating choroidal melanoma and retinoblastoma. Trans Am Ophthalmol Soc. 1973;71:171-185.

7. Shields CL, Mashayekhi A, Dai VK, Materin MA, Shields JA. Optical coherence tomographic findings of combined hamartoma of the retina and retinal pigment epithelium. Arch Ophthalmol. 2005; 123(12):1746-1750. 
8. McDonald HR, Abrams GW, Burke JM, Neuwirth J. Clinico-pathologic results of vitreous surgery for epiretinal membranes in patients with combined retinal and retinal pigment epithelial hamartomas. Am $J$ Ophthalmol. 1985;100(6):806-813.

9. Sappenfeld DL, Gitter KA. Surgical intervention for combined hamartoma of the retina and retinal pigment epithelial hamartoma. Retina. 1990;10(2):119-124.
10. Shields CL, Thangappan A, Hartzell Kimberly, Valente P, Pirondini C, Shields JA. Combined hamartoma of the retina and retinal pigment epithelium in 77 consecutive patients. Visual outcome based on macular versus extramacular tumor location. Ophthalmology. 2008; 115(12):2246-2252.

11. Holz FG. Autofluorescence imaging of the macula. Ophthalmology. 2001;98(1):10-18.

\section{Publish your work in this journal}

Clinical Ophthalmology is an international, peer-reviewed journal covering all subspecialties within ophthalmology. Key topics include: Optometry; Visual science; Pharmacology and drug therapy in eye diseases; Basic Sciences; Primary and Secondary eye care; Patient Safety and Quality of Care Improvements. This journal is indexed on

\section{Dovepress}

PubMed Central and CAS, and is the official journal of The Society of Clinical Ophthalmology (SCO). The manuscript management system is completely online and includes a very quick and fair peer-review system, which is all easy to use. Visit http://www.dovepress.com/ testimonials.php to read real quotes from published authors. 